\title{
Tip-Over Stability Analysis of a Pelvic Support Walking Robot
}

\author{
Yawei Han, ${ }^{1}$ Shuai Guo $\mathbb{D}^{1},{ }^{1}$ Leigang Zhang, ${ }^{1}$ Fengfeng (Jeff) $\mathrm{Xi}^{2}{ }^{2}$ and Weiwei $\mathrm{Lu}^{3}$ \\ ${ }^{1}$ Department of Mechatronic Engineering and Automation, Shanghai University, Shanghai, China \\ ${ }^{2}$ Department of Aerospace Engineering, Ryerson University, Toronto, Canada \\ ${ }^{3}$ Shanghai Testing \& Inspection Institute for Medical Devices, Shanghai, China \\ Correspondence should be addressed to Shuai Guo; guoshuai@shu.edu.cn
}

Received 12 August 2019; Revised 21 November 2019; Accepted 23 December 2019; Published 7 February 2020

Guest Editor: Ludovico Minati

Copyright (C) 2020 Yawei Han et al. This is an open access article distributed under the Creative Commons Attribution License, which permits unrestricted use, distribution, and reproduction in any medium, provided the original work is properly cited.

\begin{abstract}
Discussed in this paper is the tip-over stability analysis of a pelvic support walking robot. To improve the activities of daily living (ADL) in hemiplegic patients, a pelvic support walking robot is proposed to help patients facilitating their rehabilitation. During the gait training with the robot, the abnormal man-machine interaction forces may lead to the tip-over of the robot, which is not beneficial to the rehabilitation process. A new method is proposed to predict the possibility of tipping over and evaluate the stability of the robot based on statics model, dynamics model, and zero-moment point (ZMP) theory. Through the interaction forces and moments analysis with static case, the safe point (ZMP) is studied, and the influence factors of force/moment are analyzed by dynamics case. An optimization algorithm based on the genetic algorithm (GA) is proposed to reduce the risk of tipping over. The simulation results show that the optimization algorithm can keep the robot from tipping over when the interaction forces exceed the safety threshold.
\end{abstract}

\section{Introduction}

As a result of the acceleration of population aging, the World Health Organization reported over 17 million confirmed cases of stroke in 2016 [1]. Most stroke patients suffer from lower-extremity motor dysfunction after surgery, which severely affects the ADL of the patient. However, $~ 70-80 \%$ of patients can benefit from the timely and effective rehabilitation process and can restore the motor function and balance function. During the gait training, the fall prevention and safe training environment are important. It is necessary to analyze the safety of the robot by the tip-over stability analysis.

In recent years, many universities, research institutes, and hospitals have developed mechanical equipment that can help the patients with the gait training. The Lokomat [2] is a highly automated suspension lower-limb rehabilitation robot that connects the lower-extremity exoskeleton system to the suspension weight reduction device through a four-bar mechanism. The robot is stationary and has little possibility of tipping over. The
Andago [3] assisted rehabilitation robot developed by HOCOMA in Switzerland reduces the load on the lower limbs during the gait training by suspending weight loss on the pelvis. This suspension weight loss has a great influence on the coordinated movement of the upper and lower limbs. The designers avoid the tip-over by adding counterweight to the moving platform. The KineAssist [4] robot uses a pelvic support device with a moving platform to realize the movement of the patient, but the rotation center of the chassis is not on the same vertical line with the rotation center of the human body, directly affecting the patient's steering comfort. Carleton University developed a mobile limb training robot called GaitEnable [5]. This robot is smaller and lighter than KineAssist. It is a combination of a mobile lower-limb training robot and a walking robot. GaitEnable step trainer is omnidirectional. The device controls the support polygon of the robot, the position of the pelvis, and the posture of the robot. The GaitEnable and KineAssist are equipped with universal wheels to maintain stability via the change of the center of mass. 
During the gait training, the man-machine interaction forces may exceed the normal values. There is a risk that the robot will tip over because the forces acting on the robot are beyond the safety threshold, causing potential harm to the patients. Based on this concerning, the tip-over stability of the robot should be analyzed to study the threshold of the interaction forces and the effective ways to stabilize the robot.

In this paper, the tip-over stability of a pelvic support walking robot, as shown in Figure 1, is analyzed: (1) the analysis of the safe range of interaction forces; (2) the analysis of the influence factors of tipping over; (3) the study of the optimization algorithm based on ZMP and GA.

\section{System Description}

The mechanical structure diagram of the robot is shown in Figure 2. Combining the revolute pair and the prismatic pair, the robot can realize the six degrees of freedom of motion in space: flexion and extension in the sagittal plane; adduction and abduction in the coronal plane; internal rotation and external rotation on the horizontal plane; and movement of the front, back, left, and right, and up and down. According the range of motion of healthy people $[6,7]$, the workspace of the robot is designed to be larger than the healthy people's need. Also, the rotation center of the robot should coincide with the people's rotation center to make the people more comfortable during the gait training.

The function of the robot is to provide the patients with pelvic support and help them during the gait training. This function is realized through the mobile platform (MP), the body weight support (BWS), and the pelvic-assisted mechanism (PAM). The PAM can support the patient and help them to realize the movement in cross section and the rotation around the sagittal axis, the vertical axis, and the coronal axis. The BWS can lift the pelvic support mechanism and patient to realize the movement in the vertical direction: the pelvis moves periodically along the vertical axis. With the help of the MP, the motion range of the patient can be extended throughout the environment.

As shown in Figure 2, the MP has two driving wheels installed on the rear to drive the mobile platform, and two universal wheels are installed at the front. With the differential motion of the two driving wheels, the robot can realize turning motion on the ground. And the robot can rotate around the center of two drive wheels' connection (around the $Z$-axis).

The BWS consists of a ball screw and a slider. The PAM is fixed at the slider, and the slider can move along the ball screw with low friction. The PAM can move along the $Z$-axis to adapt the motion of the pelvis.

The PAM provides support to the pelvis and can realize the motion of the pelvis. And the signals that needed to control the robot or evaluate the patient's movement are detected in this part.

The movement along the $X$-axis and $Y$-axis on the horizontal plane is realized by a four-bar mechanism as shown in Figure 2(b). The four-bar mechanism consists of four links. The rotation of $J_{2}$ will produce the movement of $J_{3}$ on the horizontal plane. A potentiometer is installed on $J_{2}$ to detect $\theta_{2}$. The movement of $J_{3}$ can be calculated: $X=l_{2} \cos \theta_{2}, Y=l_{2} \sin \theta_{2}$. In order to provide elasticity in the direction of $X$-axis and $Y$-axis, a spring damping mechanism is installed between the four links. The forces will overcome the movement along the $X$-axis and $Y$-axis and make the patient know whether their pelvis center deviates from the normal position. The elasticity also can be adjusted according to different patients' needs. The rotation of $J_{4}$ realizes the rotation around the $X$-axis (the sagittal axis $(\alpha))$. Springs are placed between $J_{4}$ and the fixed base of the pelvic support mechanism to provide elasticity around the $X$-axis. $\theta_{4}$ can be detected by the potentiometer installed on $J_{4} . P_{5}$ and $P_{6}$ are prismatic pairs. When $P_{5}$ moves forward and $P_{6}$ moves backward, the patient will realize the rotation around the $Z$-axis (the vertical axis $(\gamma)$ ). The sliders of $P_{5}$ and $P_{6}$ are located between springs to provide elasticity along the $Z$-axis. The pressure sensors detect the force from patient's rotation acting on the spring. By analyzing the forces, the direction of the rotation along the $Z$-axis can be studied. Also, the movement of $P_{5}$ and $P_{6}$ can be calculated through the force $F$ and spring stiffness $k$ : $s=F / k$. And $\theta_{5}$ can be calculated as: $\theta_{5} \approx\left(S_{5}-S_{6}\right) / D$ when $\theta_{5}$ is small enough. Two universal joints are fixed at $P_{5}$ and $P_{6}$ to realize the rotation around the $Y$-axis (coronal axis $(\beta))$.

\section{System Modeling and ZMP Theory}

3.1. Statics Modeling. As shown in Figure 2, the robot coordinate system $\left(\mathrm{O}_{0} \mathrm{X}_{0} Y_{0} Z_{0}\right)$ is fixed at the center of the connection of two driving wheels. The end-effector of the robot is the pelvis of patient, the $\mathrm{O}_{6} X_{6} Y_{6} Z_{6}$ is attached. $O_{i} X_{i} Y_{i} Z_{i}(i=1,2, \ldots, 5)$ is the joint coordinate system. The rotation center of the pelvis has to be consistent with the rotation center of the mechanism: $l_{0}=l_{1}+l_{2}+l_{3}+l_{4}$; During the gait training, the parameter $Z_{1}$ will change accordingly to realize the displacement in the $Z$-axis direction. The parallel four-bar mechanism composed of joints $J_{2}$ and $J_{3}$ realizes the $X$-axis and the displacement in the $Y$-axis direction: $X=l_{2} \cos \theta_{2}, Y=l_{2} \sin \theta_{2}$ (relative to the coordinate system $\sum 2$ ). The establishment of the positive solution matrix equation is based on the recursive formula proposed by $\mathrm{Xi}$ et al., which is as follows [8]:

$$
\begin{aligned}
& \mathbf{p}_{i}=\mathbf{p}_{0}+\sum_{i=1}^{n} \mathbf{R}_{i} \mathbf{p}_{i}^{\prime}, \\
& \mathbf{R}_{i}=\prod_{j=1}^{i} \mathbf{R}_{s j} \mathbf{R}_{m j},
\end{aligned}
$$

where

(i) $\mathbf{p}_{i}$ is the position of each joint relative to the world coordinate system;

(ii) $\mathbf{p}_{i}^{\prime}$ is the position vector;

(iii) $\mathbf{R}_{i}$ is the rotation transformation matrix; 


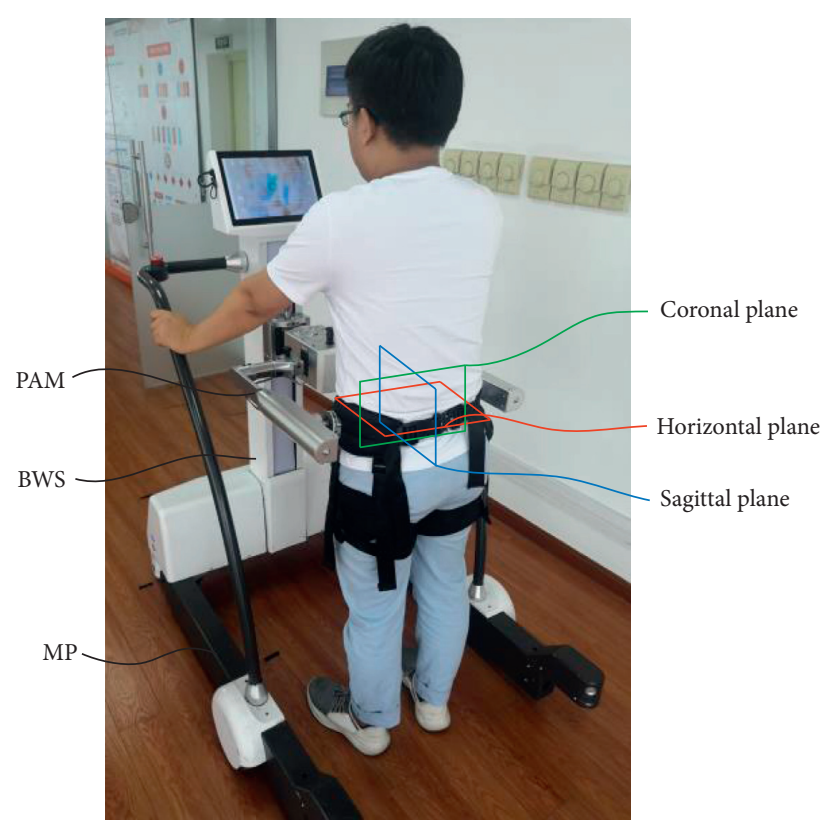

Figure 1: The components of the pelvic support walking robot. (a) The coordinate system. (b) The setting of the springs and sensors.
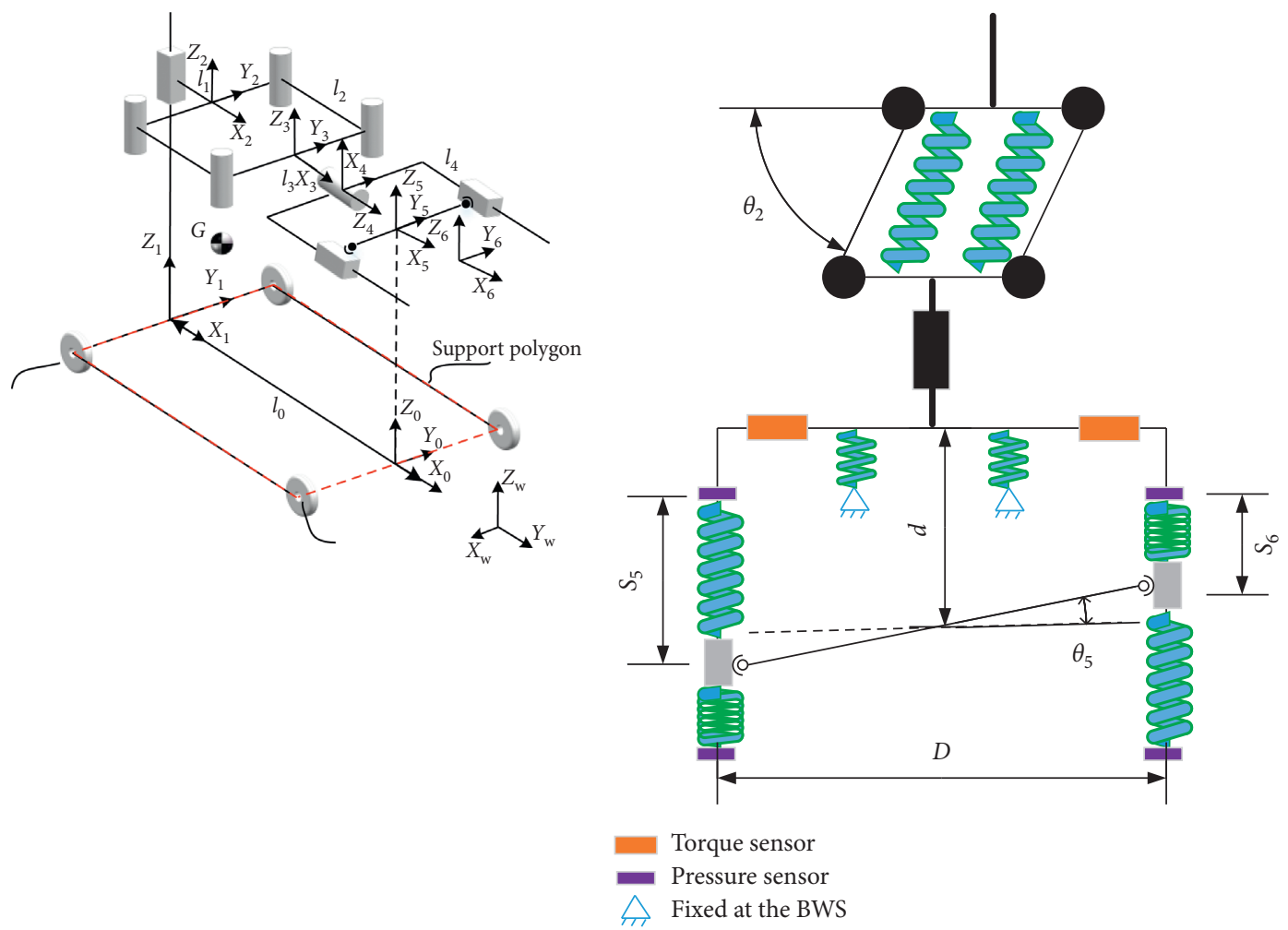

(a)

(b)

FIgURE 2: The schematic diagram of the mechanism.

(iv) $\mathbf{R}_{s j}$ is the coordinate transformation of the adjacent coordinate system;

(v) $\mathbf{R}_{m j}$ is the rotation around the current coordinate system, according to the mechanism diagram:

$$
\left[\begin{array}{ll}
\mathbf{R} & \mathbf{P} \\
0 & 1
\end{array}\right]=\left[\begin{array}{cccc}
C_{5} C_{6} & -S_{5} & C_{5} S_{6} & T_{1} \\
T_{2} & C_{4} C_{5} & T_{3} & l_{2} S_{2} \\
T_{4} & C_{5} S_{4} & T_{5} & Z_{0} \\
0 & 0 & 0 & 1
\end{array}\right]
$$


where

$C_{2}=\cos \theta_{2}, S_{2}=\sin \theta_{2}$, and so on; $T_{1}=l_{0}-l_{1}-l_{3}-$ $l_{2} C_{2}-l_{4} ; T_{2} \quad=S_{4} S_{6}+C_{4} S_{5} C_{6} ; T_{3}=C_{4} S_{5} S_{6}-S_{4} C_{6} ; T_{4}$ $=C_{6} S_{4} S_{5}+C_{4} S_{6} ; T_{5}=C_{4} C_{6}+S_{4} S_{5} S_{6}$.

$$
\left[\begin{array}{c}
v_{x} \\
v_{y} \\
v_{z} \\
\omega_{x} \\
\omega_{y} \\
\omega_{z}
\end{array}\right]=\left[\begin{array}{cccccc}
0 & -l_{2} S_{2} & 0 & 0 & 0 & 0 \\
0 & l_{2} C_{2} & 0 & 0 & 0 & 0 \\
1 & 0 & 0 & 0 & 0 & 0 \\
0 & 0 & 1 & 0 & 0 & 0 \\
0 & 0 & 0 & 0 & 0 & 1 \\
0 & 0 & 0 & \frac{1}{D} & -\frac{1}{D} & 0
\end{array}\right]\left[\begin{array}{c}
\dot{Z}_{1} \\
\dot{\theta}_{2} \\
\dot{\theta}_{4} \\
\dot{S}_{5} \\
\dot{S}_{6} \\
\dot{\theta}_{6}
\end{array}\right],
$$

where $D=0.32 \mathrm{~m}$, as shown in Figure 2, is the width of the mechanism, which hugs the pelvis.

The center of mass of the link can be viewed as at the center of figure of the link. The vector of the $i$ th link centroid position in the $\sum 0$ :

$$
\mathbf{p}_{c i}=\mathbf{p}_{i-1}+\frac{1}{2} \mathbf{p}_{i}=\mathbf{R}_{i} \mathbf{p}_{c i}^{\prime} .
$$

The centroid velocity of the $i$ th link $\dot{p}_{c i}$ can be got by derivation on both sides of the equation:

$$
\dot{p}_{c i}=\dot{R}_{i} \mathbf{p}_{c i}^{\prime}+\mathbf{R}_{i} \dot{p}_{c i}^{\prime}=\boldsymbol{\omega}_{i} \times \mathbf{p}_{c i}+\mathbf{R}_{i} \dot{p}_{c i}^{\prime} .
$$

The centroid acceleration of the $i$ th link $\ddot{p}_{c i}$ can be got by derivation on both sides of equation (5):

$$
\begin{aligned}
\ddot{p}_{c i} & =\dot{\omega}_{i} \times \mathbf{p}_{c i}+\boldsymbol{\omega}_{i} \times \dot{p}_{c i}+\dot{R}_{i} \dot{p}_{c i}^{\prime}+\mathbf{R}_{i} \ddot{p}_{c i}^{\prime} \\
& =\dot{\omega}_{i} \times \mathbf{p}_{c i}+\boldsymbol{\omega}_{i} \times\left(\boldsymbol{\omega}_{i} \times \mathbf{p}_{c i}+\mathbf{R}_{i} \mathbf{p}_{c i}^{\prime}\right)+\dot{R}_{i} \mathbf{R}_{i}^{T} \mathbf{R}_{i} \dot{p}_{c i}^{\prime}+\mathbf{R}_{i} \ddot{p}_{c i}^{\prime} \\
& =\dot{\omega}_{i} \times \mathbf{p}_{c i}+\boldsymbol{\omega}_{i} \times\left(\boldsymbol{\omega}_{i} \times \mathbf{p}_{c i}\right)+2\left(\boldsymbol{\omega}_{i} \times \mathbf{R}_{i} \mathbf{p}_{c i}^{\prime}\right)+\mathbf{R}_{i} \ddot{p}_{c i}^{\prime},
\end{aligned}
$$

where

(i) $\boldsymbol{\omega}_{i}$ is the joint angular velocity of $i$ th link;

(ii) $\mathbf{p}_{c i}$ is the vector of the $i$ th link centroid position in the $\sum 0 ;-\dot{p}_{c i}^{\prime}$ is the velocity of $i$ th link;

(iii) $\mathrm{R}_{i}$ is the transformation from $\sum 0$ to $\sum i$.

3.2. Dynamics Modeling. As the movement of the pelvis has great influence on the robot's tip-over stability, it is necessary to establish a dynamics model to research the motion parameters' effect on the robot's risk of tipping over.

According to $\mathrm{Xi}$ et al., the force and moment acting on the $i$ th joint:

$$
\mathbf{w}_{i}=\mathbf{M}_{i} \dot{t}_{i J}+\mathbf{B}_{i}+\mathbf{H}_{i i+1} \mathbf{w}_{i+1}
$$

where

(i) $\mathbf{w}_{i}=\left[\begin{array}{ll}\mathbf{f}_{i J} & \mathbf{m}_{i J}\end{array}\right]$ consists of the force and moment of the $i$ th joint; (ii) $\mathbf{M}_{i}=\left[\begin{array}{cc}\mathbf{m}_{i} 1 & \mathbf{m}_{i} \widetilde{p}_{i c}^{T} \\ \mathbf{m}_{i} \widetilde{p}_{i c} & \mathbf{I}_{i}\end{array}\right]$ is the generalized mass matrix of the $i$ th link;

(iii) $\widetilde{p}_{i c}$ is the skew symmetric matrix of centroid vector of the $i$ th joint;

(iv) $\mathbf{I}_{i}$ is the inertia tensor of each link;

(v) $\dot{t}_{i J}=\left[\begin{array}{c}\ddot{p}_{c i}-\mathbf{g} \\ \boldsymbol{\alpha}_{i}\end{array}\right]$ is the acceleration of the $i$ th link;

(vi) $\mathbf{B}_{i}=\left[\begin{array}{c}m_{i} \boldsymbol{\omega}_{i} \times\left(\boldsymbol{\omega}_{i} \times \mathbf{p}_{i c}\right) \\ \boldsymbol{\omega}_{i} \times\left(\mathbf{I}_{i} \boldsymbol{\omega}_{i}\right)\end{array}\right]$ consists of the centrifugal force and gyro moment;

(vii) $\omega_{i}$ is the angular velocity of the $i$ th joint;

(viii) $\mathbf{p}_{i c}$ is the vector pointing from the center of the $i$ th link to the $i$ th joint;

(ix) $\mathbf{H}_{i i+1}=\left[\begin{array}{cc}1 & 0 \\ \widetilde{p}_{i} & 1\end{array}\right]$ is the transformation matrix from $i+1$ th joint to $i$ th joint;

(x) $\mathbf{p}_{i}$ is the vector pointing from joint $i$ to the tip;

(xi) $\tilde{p}_{i}$ is the antisymmetric matrix of $\mathbf{p}_{i}$.

All the forces and moments acting on the machine converted to joint $i$ can be written as:

$$
\begin{aligned}
\mathbf{w}_{i}= & \mathbf{M}_{i} \dot{t}_{i J}+\sum_{k=i+1}^{5}\left(\prod_{j=k+1}^{k} H_{j-1, j}\right) \mathbf{M}_{k} \dot{t}_{k J}+\mathbf{B}_{i} \\
& +\sum_{k=i+1}^{5}\left(\prod_{j=k+1}^{k} \mathbf{H}_{j-1, j}\right) B_{k}+\left(\prod_{j=i+1}^{5} \mathbf{H}_{j-1, j}\right) \mathbf{w}_{p},
\end{aligned}
$$

where $w_{p}$ is the forces and moments between patient and robot.

3.3. ZMP Theory. The zero-moment point theory [9] is used to evaluate the tip-over stability by comparing the relative positional relationship between the ZMP and the support polygon. The component of the man-machine interaction forces and moments in the horizontal plane needs to be balanced by the friction and the friction torque:

$$
\begin{array}{r}
(\overrightarrow{O P} \times \vec{R})^{H}+\overrightarrow{O G} \times m_{s} g+\mathbf{M}_{A}^{H}+\left(\overrightarrow{O A} \times \mathbf{F}_{A}\right)^{H}=0, \\
\mathbf{R}+\mathbf{F}_{A}+m_{s} \mathbf{g}=0,
\end{array}
$$

where

(i) $\overrightarrow{O P}$ is the vector from the $\sum 0$ to the acting point of the friction force;

(ii) $\vec{R}$ is the friction force from the ground and can be calculated from equation (9);

(iii) $G$ is the center of gravity of the robot.

The force $\mathbf{F}_{A}$ and moment $\mathbf{M}_{A}$ equals to the forces and moments above the $\sum 0$. 


$$
\begin{aligned}
\mathbf{R}_{x} & =-\left(\mathbf{F}_{A x}+m_{s} \mathbf{g}_{x}\right), \\
\mathbf{R}_{y} & =-\left(\mathbf{F}_{A y}+m_{s} \mathbf{g}_{y}\right), \\
\mathbf{R}_{z} & =-\left(\mathbf{F}_{A z}+m_{s} \mathbf{g}_{z}\right) .
\end{aligned}
$$

The position of the point $P\left(P_{x}, P_{y}\right)$ (zero-moment point) can be calculated as:

$$
\begin{aligned}
& P_{x}=\frac{\left(\overrightarrow{O G} \times m_{s} \mathbf{g}+\mathbf{M}_{A}^{H}+\left(\overrightarrow{O A} \times \mathbf{F}_{A}\right)^{H}\right)_{y}}{\mathbf{R}_{z}}, \\
& P_{y}=\frac{\left(\overrightarrow{O G} \times m_{s} \mathbf{g}+\mathbf{M}_{A}^{H}+\left(\overrightarrow{O A} \times \mathbf{F}_{A}\right)^{H}\right)_{x}}{\mathbf{R}_{z}} \\
& P_{z}=0 .
\end{aligned}
$$

The support polygon is made up of a rectangle. Once the $P$ is out of the rectangle, the robot will lose dynamic equilibrium and tip over.

\section{System Simulation and Optimization}

The simulations are carried out to analyze the possibility of tipping over of the robot. The input is man-machine interaction forces. These forces can be measured and calculated:

$\mathbf{F}_{x}$ is the force generated by the movement of the patient along the $X$-axis. $\mathbf{F}_{x}$ can be measured by the pressure sensors as shown in Figure 2(b).
$\mathbf{F}_{y}$ is the force generated by the movement of the patient along the $Y$-axis. $\mathbf{F}_{y}$ can be calculated as follows:

$$
\mathbf{F}_{y}=\Delta y K_{23} \text {, }
$$

where $\Delta y$ is the movement along the $Y$-axis, $K_{23}$ is the spring damping stiffness between joint 2 and joint $3 . K_{23}$ can be calculated through experiment. In this robot, $K_{23}=3.9 \mathrm{~N} / \mathrm{mm}$.

$\mathbf{F}_{z}$ is the force generated by the movement of the patient along the $Z$-axis. $\mathbf{F}_{z}$ can be measured and calculated by the torque sensors as shown in Figure 2(b).

$$
\mathbf{F}_{z}=\frac{T}{d},
$$

where $T$ is the torque measured by the torque sensors. $d$ is the distance between the torque sensors and the center of the pelvis.

The support polygon is a rectangle: $L * W(1.03 * 0.7 \mathrm{~m})$. The coinciding of the round reaction force acting point $(P)$ with the center of the rectangle can be seen as the safest condition of the robot. Evaluation function $Q$ can describe the relation between the two points. If $P$ coincides with the center of the support polygon, the function $Q$ is 1 . When $Q$ is closer to 1 , the robot is safer and less likely to tip over. When $Q$ is closer to 0 , the robot is more likely to tip over. If $P$ exceeds the support polygon boundary, the function $Q$ will be negative.

$$
Q= \begin{cases}\frac{\min \left(\left|P_{x}\right|,\left|P_{x}+(L / 2)\right|\right)}{L}+\frac{\min \left(\left|P_{y}-(W / 2)\right|,\left|P_{y}+(W / 2)\right|\right)}{W}, & \text { when the } \operatorname{ZMP}\left(P_{x}, P_{y}\right) \text { is inside the support polygon, } \\ -1, & \text { when the } \operatorname{ZMP}\left(P_{x}, P_{y}\right) \text { is outside the support polygon. }\end{cases}
$$

4.1. Static Simulation. An experiment is designed to study the range of $\mathbf{F}_{x}, \mathbf{F}_{y}$, and $\mathbf{F}_{z}$, when the subject falls over and leans on the machine. As shown in Figure 3, there are four cases when the subject leans on the robot and the 8 subjects involved with different height and weight. The result is shown in Table 1. The static calculation model can be got by making $\boldsymbol{\omega}_{i}=0, \ddot{p}_{c i}=0$, and $\boldsymbol{\alpha}_{i}=0$ in the dynamic model. And so on, $\mathbf{B}_{i}=0$ and $\dot{t}_{i J}=\left[\begin{array}{c}-\mathbf{g} \\ 0\end{array}\right]$. The force and moment acting on the $i$ th joint in the static model can be written as:

$$
\mathbf{w}_{i}=\mathbf{M}_{i} \dot{t}_{i J}+\sum_{k=i+1}^{5}\left(\prod_{j=k+1}^{k} \mathbf{H}_{j-1, j}\right) \mathbf{M}_{k} \dot{t}_{k J}+\left(\prod_{j=i+1}^{5} \mathbf{H}_{j-1, j}\right) \mathbf{w}_{p} .
$$

In the statics model, the parameter $Z_{0}$ is the height of the pelvis, and the range of $Z_{1}$ is from $0.55 \mathrm{~m}$ to $1.1 \mathrm{~m}$. The simulations with different $Z_{1}$ are carried out. The results in Figure 4 show that the higher the $Z_{1}$ is, the more risk the robot with the same interaction force.

In order to analyze the safe range of the input forces, make $\quad \mathbf{F}_{x}: \quad-450 \mathrm{~N} \sim 300 \mathrm{~N}, \quad \mathbf{F}_{y}:-200 \mathrm{~N} \sim 300 \mathrm{~N}, \quad \mathbf{F}_{z}$ : $-350 \mathrm{~N} \sim 350 \mathrm{~N}$. Within the range of forces set, calculate $Q$ with each force synthesized by $\mathbf{F}_{x}, \mathbf{F}_{y}$, and $\mathbf{F}_{z}$, and $Z_{1}=1.1 \mathrm{~m}$. The calculation result is shown in Figure 5, and the force safety range: $\mathbf{F}_{X Y Z}=\left[\mathbf{F}_{X}, \mathbf{F}_{Y}, \mathbf{F}_{Z}\right]^{T}$ is as follows:

$$
\mathbf{F}_{X Y Z}=\left\{\begin{array}{l}
\mathbf{F}_{Z} \leq 3.1 \mathbf{F}_{Y}+320, \mathbf{F}_{Z} \leq 1.2 \mathbf{F}_{X}+180 \\
\text { s.t. } 0 \leq \mathbf{F}_{Y} \leq 200,0 \leq \mathbf{F}_{X} \leq 140 \\
\mathbf{F}_{Z} \leq-3.1 \mathbf{F}_{Y}+320, \mathbf{F}_{Z} \leq 1.2 \mathbf{F}_{X}+180 \\
\text { s.t. }-200 \leq \mathbf{F}_{Y}<0,-400 \leq \mathbf{F}_{X} \leq 0
\end{array}\right.
$$

The points with light color means that with the effect of the forces the point represents, the ZMP: $P$ is within the 


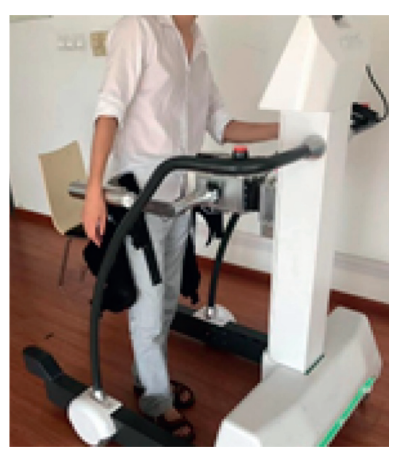

(a)

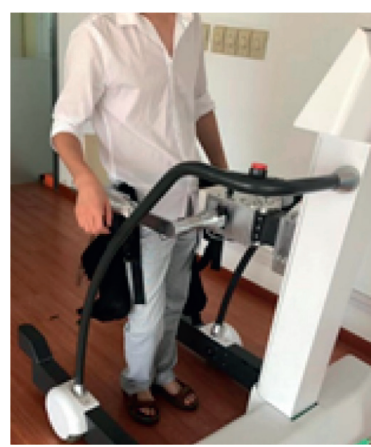

(b)

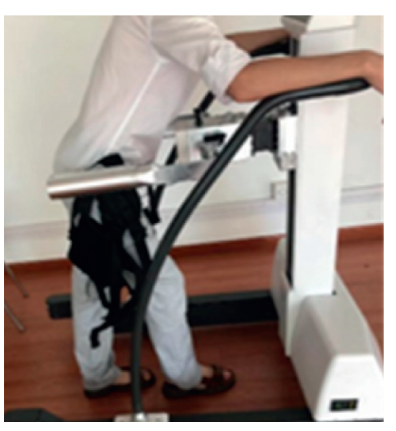

(c)

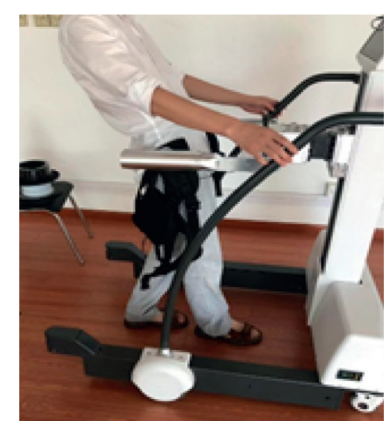

(d)

Figure 3: The four cases that the subject leans on the robot. (a) The subject leans on the machine to the left; (b) the subject leans on the machine to the right; (c) the subject leans on the machine to the forward; (d) the subject leans on the machine to the backward.

Table 1: The force along the $X$-axis and $Y$-axis when the patient falls over.

\begin{tabular}{lcccccc}
\hline Subject & $\begin{array}{c}\text { Height } \\
(\mathrm{cm})\end{array}$ & $\begin{array}{c}\text { Weight } \\
(\mathrm{kg})\end{array}$ & $\begin{array}{c}+F_{x} \\
(\mathrm{~N})\end{array}$ & $\begin{array}{c}-F_{x} \\
(\mathrm{~N})\end{array}$ & $\begin{array}{c}+F_{\mathrm{y}} \\
(\mathrm{N})\end{array}$ & $\begin{array}{c}-F_{y} \\
(\mathrm{~N})\end{array}$ \\
\hline S1 & 178 & 73 & 130 & 140 & 130 & 130 \\
S2 & 170 & 78 & 160 & 165 & 155 & 130 \\
S3 & 178 & 65 & 130 & 130 & 120 & 125 \\
S4 & 173 & 55 & 90 & 90 & 85 & 75 \\
S5 & 173 & 60 & 140 & 130 & 110 & 90 \\
S6 & 173 & 62 & 100 & 110 & 100 & 110 \\
S7 & 173 & 68 & 120 & 120 & 120 & 120 \\
S8 & 169 & 63 & 130 & 140 & 100 & 110 \\
Average & 173 & 66 & 125 & 128 & 115 & 111 \\
\hline
\end{tabular}

support polygon. If the color is blue, the robot will tip over and the patient is in danger.

The analysis of the tip-over stability in the static condition provides reference for the analysis with dynamics model. The impact factors such as $\boldsymbol{\omega}_{i}, \boldsymbol{\alpha}_{i}, \ddot{p}_{c i}$, and so on will be considered, and a control system based on genetic algorithm (GA) [10,11] is used to optimize the tip-over stability of the robot.

4.2. Dynamic Simulation. The simulation with dynamic model considers the effect from the $\omega_{i}$ (joint angular velocity) on the analysis of robot. The consideration of $\boldsymbol{\omega}_{i}$ makes $\mathbf{I}_{i}$ (the inertia tensor of $i$ th link) become an important influencing factor:

$$
\begin{aligned}
\mathbf{B}_{i} & =\left[\begin{array}{c}
m_{i} \boldsymbol{\omega}_{i} \times\left(\boldsymbol{\omega}_{i} \times \mathbf{p}_{i c}\right) \\
\boldsymbol{\omega}_{i} \times\left(\mathbf{I}_{i} \mathbf{\omega}_{i}\right)
\end{array}\right], \\
\mathbf{M}_{i} \dot{t}_{i J} & =\left[\begin{array}{cc}
m_{i} 1 & m_{i} \widetilde{p}_{i c}^{T} \\
m_{i} \widetilde{p}_{i c} & \mathbf{I}_{i}
\end{array}\right]\left[\begin{array}{c}
\ddot{p}_{c i}-\mathbf{g} \\
\boldsymbol{\alpha}_{i}
\end{array}\right] .
\end{aligned}
$$

As can be seen from equations (18) and (19), the larger the $\boldsymbol{\omega}_{i}$ and $\mathbf{I}_{i}$ are, the larger the $\boldsymbol{\omega}_{i}$ is. According to the quality of each link and the motion pattern of each joint, $\boldsymbol{\omega}_{2}$ and $\boldsymbol{\omega}_{4}$ may have great influence on the tip-over stability of the robot. The results of the simulation about $\boldsymbol{\omega}_{2}$ and $\boldsymbol{\omega}_{4}$ are shown in Figures 6 and 7 . Although the variation range of $\omega_{2}$ and $\omega_{4}$ is four times than the normal value, the results show that $\omega_{2}$ and $\omega_{4}$ have little influence on the forces and moments of last joint.

4.3. Optimization. According to the analysis above, the height of the pelvis: $Z_{1}$ has great influence on the tip-over stability of the robot. And other parameters such as $\omega_{2}$ and $\omega_{4}$ have little influence on the robot. Based on this, in the progress of the optimization, $Z_{1}$ and the movement along $X$ axis: $\Delta X$ will be adjusted to look for the best combination of $Z_{1}$ and $\Delta X$ to make $Q$ nearest to 1 .

The range of interaction force: $\mathbf{F}_{n x y z}=\left[\mathbf{F}_{n x}, \mathbf{F}_{n y}, \mathbf{F}_{n z}\right]$ when healthy people walks with the robot normally can be obtained from the study by Ji et al. [12].

According to the analysis in the static simulation, the safe range of the interaction force: $\mathbf{F}_{X} \mathbf{F}_{Y}$ and $\mathbf{F}_{Z}$ is studied. Also, when the patient loses support and falls over, the interaction force: $\mathbf{F}_{x y z}=\left[\mathbf{F}_{x}, \mathbf{F}_{y}, \mathbf{F}_{z}\right]$ is beyond the safe range. In order to make sure the robot is safer during using, safety factor $H$ is proposed to calculate the safety threshold of the interaction force:

$$
\mathbf{F}_{X Y Z}>\mathbf{F}_{s x y z}=\frac{\mathbf{F}_{X Y Z}}{H}>\mathbf{F}_{n x y z} .
$$

An optimization algorithm based on genetic algorithm (GA) is proposed to prevent the robot from tipping over and make the evaluation function $Q$ nearest to 1 . The control flow chart is shown in Figure 8. When the interaction force is beyond $\mathbf{F}_{s x y z}$, GA will calculate the optimal $\Delta Z_{1}$ and $\Delta X$. GA calculates the $Q$ with different combinations of $Z_{1}$ and $\Delta X$, and chooses $Z_{Q 1}$ and $\Delta X_{Q}$ as the best combination when $Q$ is the maximum. And the signals will be sent to the motors to adjust the height of the pelvic and the position of the support polygon.

$$
\begin{array}{rc}
\underbrace{\text { Maximize }}_{Z_{Q 1}, \Delta X_{Q}, Q} & \mathrm{GA}\left(Z_{1}, \Delta X, Q\right) \\
\text { s.t. } & 0.55 \leq Z_{1} \leq 1.1, \\
& -0.3 \leq \Delta X \leq 0.3 .
\end{array}
$$




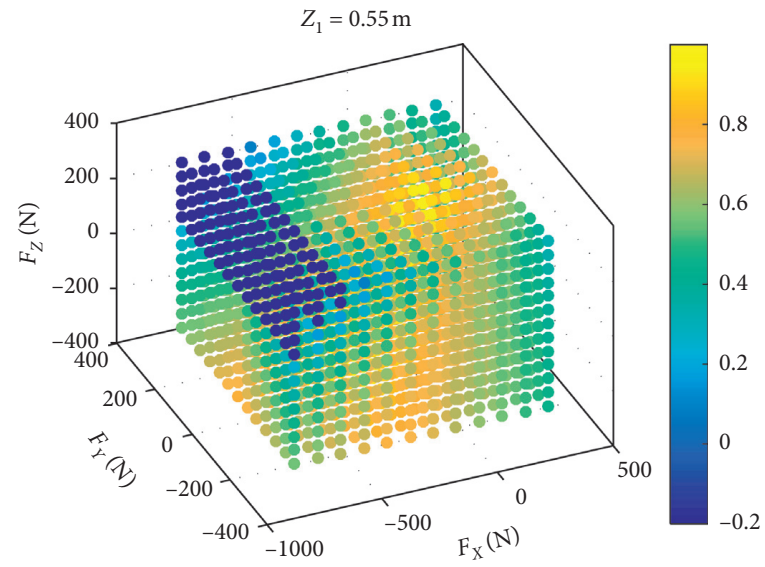

(a)

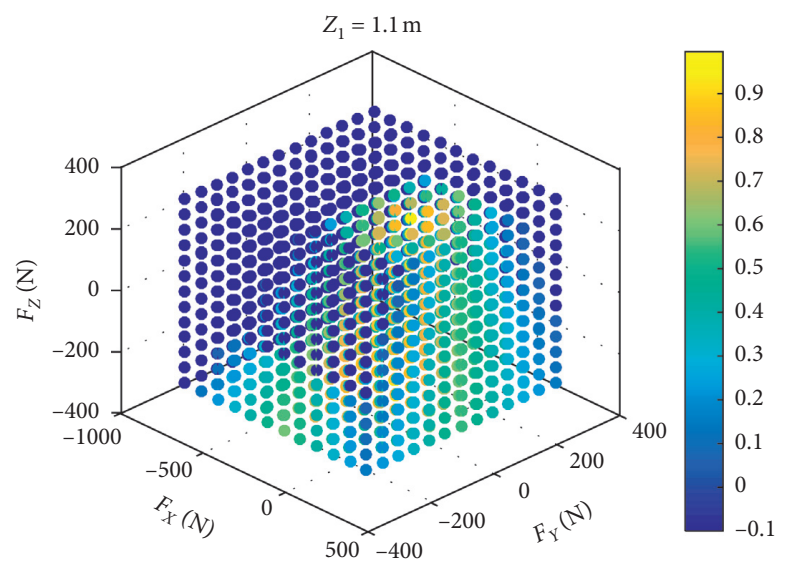

(b)

FIGURE 4: The relationship between the tip-over stability of the robot and the height of the pelvis: $Z_{1}$. Each point represents an interaction force: $\mathbf{F}_{\mathrm{xyz}}$. The points with light color mean the robot will not tip over, and the points with blue color mean the robot will tip over. (a) The number of the safe points is 1898 with the $Z_{1}=0.55 \mathrm{~m}$. (b) The number of the safe points is 1225 with the $Z_{1}=1.1 \mathrm{~m}$.

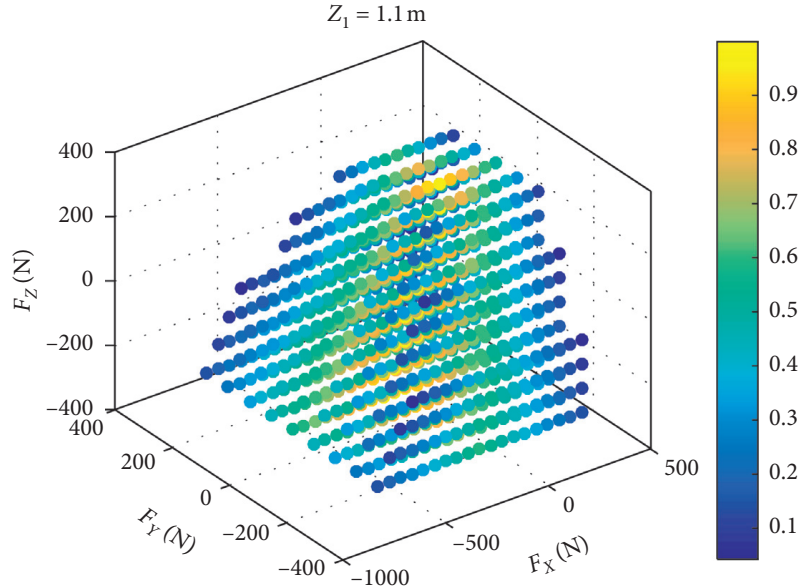

Figure 5: The safe range of $F_{X Y Z}$ with $Z_{1}=1.1 \mathrm{~m}$.
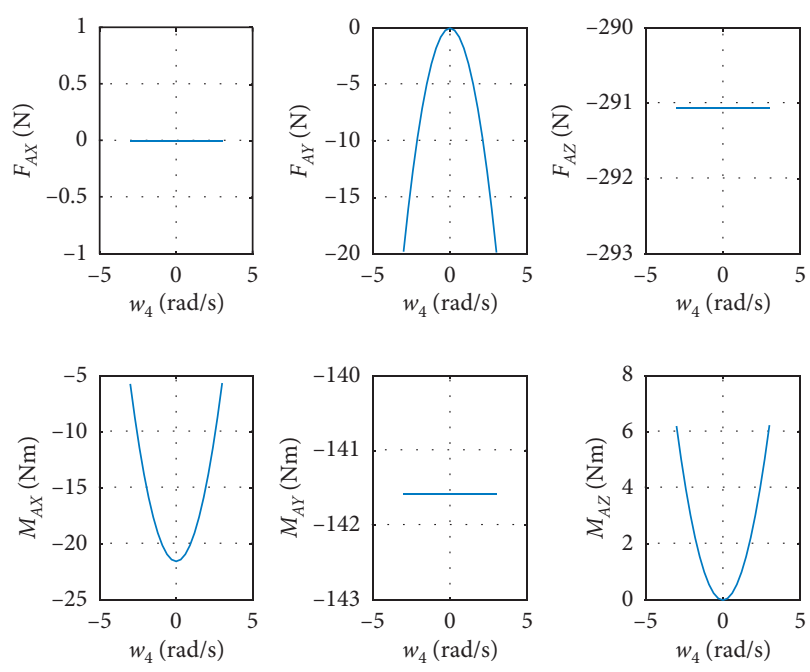

FIgURE 6: The influence of $\omega_{2}$ on the forces: $F_{A x}, F_{A y}$ and $F_{A z}$ and the moments: $M_{A x}, M_{A y}$ and $M_{A z}$.
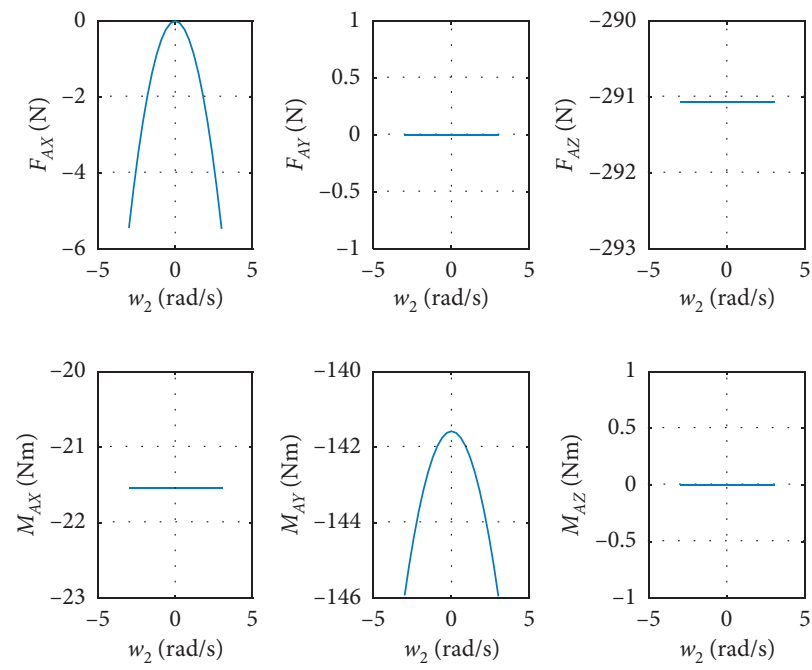

Figure 7: The influence of $\omega_{4}$ on the forces: $F_{A x}, F_{A y}$ and $F_{A z}$ and the moments: $M_{A x}, M_{A y}$ and $M_{A z}$.

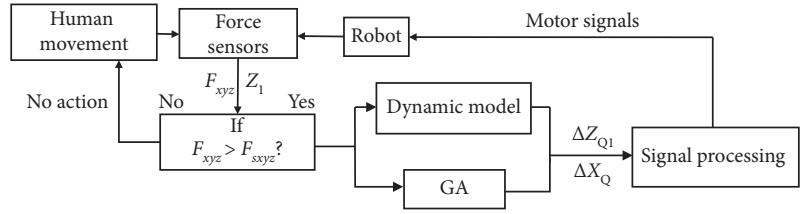

FIgURE 8: The control flow of the optimization algorithm.

4.4. Simulation. In order to ensure the safety of the subject, a simulation is carried to instead of an experiment. The results are shown in Figures 9 and 10. In Figure 9, without the optimization algorithm, the distribution of the zeromoment points: $P$ is throughout the support polygon. And in Figure 10, with the optimization, all the points are distributed around the center of the center of the support polygon. 


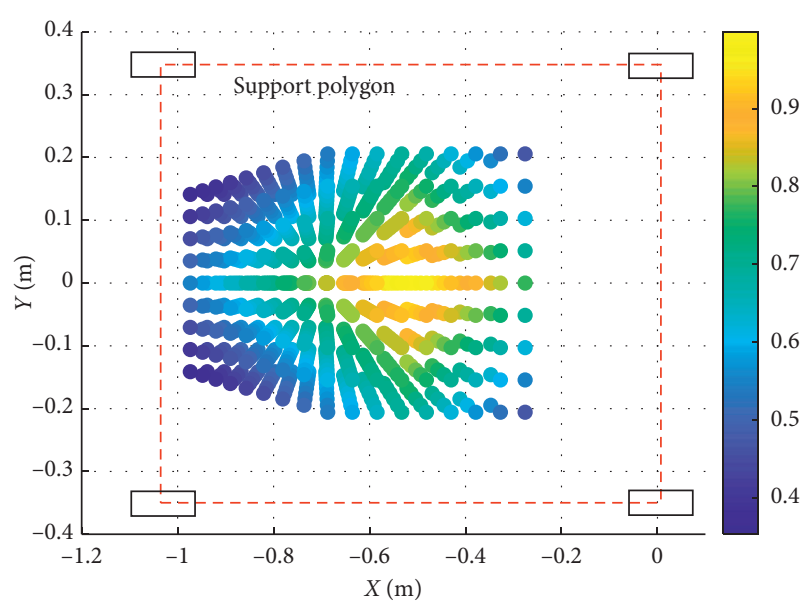

Figure 9: The distribution of $P(\mathrm{ZMP})$ without the optimization algorithm.

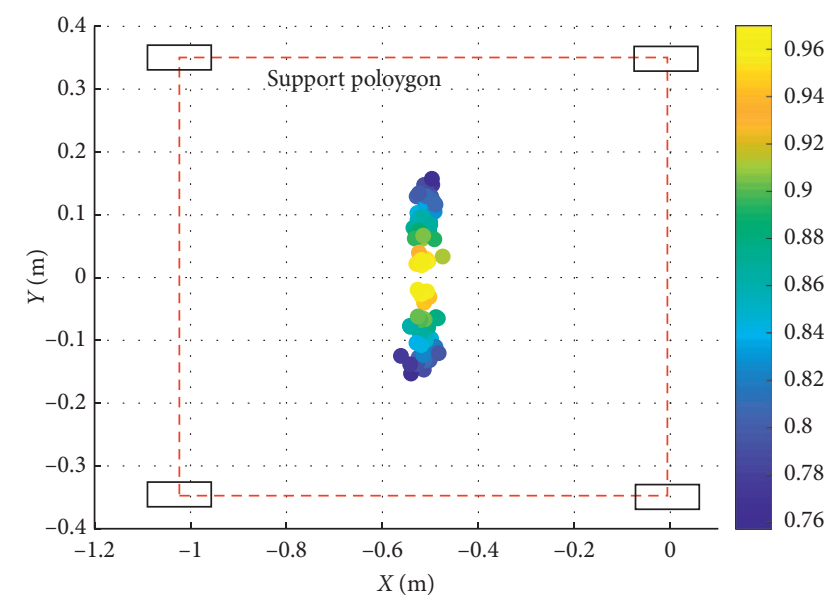

Figure 10: The distribution of $P(\mathrm{ZMP})$ with the optimization algorithm.

4.5. Data Analysis and Discussion. The tip-over stability of the pelvic support walking robot is analyzed based on the statics model, the dynamics model, and the ZMP theory. Figure 3 shows the results of two simulations carried out with $\quad \mathbf{F}_{x}: \quad-800 \mathrm{~N} \sim 300 \mathrm{~N}, \quad \mathbf{F}_{y}: \quad-300 \mathrm{~N} \sim 300 \mathrm{~N}, \quad \mathbf{F}_{z}$ : $-350 \mathrm{~N} \sim 350 \mathrm{~N}$. In Figure $4(\mathrm{a})$, with $Z_{1}=0.55 \mathrm{~m}$, the most points' $Q$ is positive with light color. The number of safe points is 1898. In Figure 4(b), with $Z_{1}=1.1 \mathrm{~m}$, half of the points' $Q$ is negative. The number of safe points is 1225 . The results show that the height of the pelvis: $Z_{1}$ has great influence on the tip-over stability of the robot. The safe range of the interaction forces is studied in Figure 5 with $Z_{1}=1.1 \mathrm{~m}$. The safe range of $\mathbf{F}_{y}$ is symmetric around the $Y$ axis from $-200 \mathrm{~N}$ to $200 \mathrm{~N}$ for that the robot is symmetry about the $x o z$ plane. The safe range of $\mathbf{F}_{x}$ is from $-400 \mathrm{~N} \sim 140 \mathrm{~N}$ for that the center of mass position is at the front of the robot. The safe range of $\mathbf{F}_{z}$ is a function of $\mathbf{F}_{x}$ and $\mathbf{F}_{y}$ as shown in (17). The influence of $\boldsymbol{\omega}_{2}$ and $\boldsymbol{\omega}_{4}$ is analyzed, and the results are shown in Figures 6 and 7. It shows that the influence of $\omega_{2}$ and $\omega_{4}$ is not so obvious, for that the max variation of $\mathbf{F}_{A}$ and $\mathbf{M}_{A}$ is $15 \mathrm{~N}$ and $20 \mathrm{~N} \cdot \mathrm{m}$, respectively. The optimization based on the GA and the influence factors: $Z_{1}$ and $\Delta X$ is proven to be effective in Figures 9 and 10. In Figure 9, without the optimization algorithm, the $P(\mathrm{ZMP})$ is distributed throughout the support polygon. Some points' $Q$ is close to 0.3 , and the position is close to the boundary of the support polygon. With the interaction forces, these dangerous points represent the robot is likely to tip over. In Figure 10 , the $Q$ of these points is from 0.76 to 1 , and the distribution of these points is limited to a rectangle: $X$ : $-0.58 \sim-0.48 ; Y:-0.16 \sim 0.16$. It means that with the optimization algorithm, the points whose interaction forces are beyond the safe range: $\mathbf{F}_{s x y z}$ are focused around the center of the support polygon with the adjustments of $Z_{1}$ and $\Delta X$.

\section{Conclusions}

The tip-over stability analysis of a pelvic support walking robot was introduced in this paper, and an optimization algorithm was proposed to optimize the tip-over stability of the robot. The influence of the height of the pelvis and the safe range of the interaction forces were studied through the simulation with statics model. With the dynamics model, it was proven that the joint angular velocity such as $\omega_{2}$ and $\omega_{4}$ have little influence on the forces and moments of the last joint. An optimization algorithm based on the dynamics model and the GA was proposed to optimize the tip-over stability when the interaction forces are beyond the safe range. Simulations were carried to verify the effectiveness of the optimization system. The follow-up work will be concentrated on the improvement of the optimization algorithm to reduce the running time of the algorithm. Also, the counterweight of the robot can be optimized to improve the tip-over stability.

\section{Appendix}

\section{Rotation Matrix}

The rotation matrix referred in 3.1 kinematics modeling is shown as:

$$
\begin{aligned}
& \mathbf{R}_{s 0}=\left[\begin{array}{lll}
1 & 0 & 0 \\
0 & 1 & 0 \\
0 & 0 & 1
\end{array}\right] ; \mathbf{P}_{0}^{\prime}=\left[\begin{array}{c}
-l_{0} \\
0 \\
0
\end{array}\right] ; \mathbf{R}_{s 1}=\left[\begin{array}{ccc}
1 & 0 & 0 \\
0 & 1 & 0 \\
0 & 0 & 1
\end{array}\right] ; \\
& \mathbf{P}_{1}^{\prime}=\left[\begin{array}{c}
Z_{1} \\
0 \\
Z_{0}
\end{array}\right] ; \mathbf{R}_{s 2}=\left[\begin{array}{ccc}
1 & 0 & 0 \\
0 & 1 & 0 \\
0 & 0 & 1
\end{array}\right] ; \mathbf{P}_{2}^{\prime}=\left[\begin{array}{c}
l_{2} C_{2} \\
l_{2} S_{2} \\
0
\end{array}\right] ; \\
& \mathbf{R}_{s 3}=\left[\begin{array}{lll}
1 & 0 & 0 \\
0 & 1 & 0 \\
0 & 0 & 1
\end{array}\right] ; \mathbf{P}_{3}^{\prime}=\left[\begin{array}{l}
l_{3} \\
0 \\
0
\end{array}\right] ; \mathbf{R}_{s 4}=\left[\begin{array}{ccc}
0 & 0 & 1 \\
1 & 0 & 0 \\
0 & 1 & 0
\end{array}\right] ; \\
& \mathbf{R}_{m 4}=\left[\begin{array}{ccc}
C 4 & -S 4 & 0 \\
S 4 & C 4 & 0 \\
0 & 0 & 1
\end{array}\right] ; \mathbf{P}_{4}^{\prime}=\left[\begin{array}{l}
0 \\
0 \\
l_{4}
\end{array}\right] ; \mathbf{R}_{s 5}=\left[\begin{array}{ccc}
1 & 0 & 0 \\
0 & 0 & 1 \\
0 & -1 & 0
\end{array}\right] ; \\
& \mathbf{R}_{m 5}=\left[\begin{array}{ccc}
C 5 & -S 5 & 0 \\
S 5 & C 5 & 0 \\
0 & 0 & 1
\end{array}\right] ; \mathbf{P}_{5}^{\prime}=\left[\begin{array}{l}
0 \\
0 \\
0
\end{array}\right] ; \mathbf{R}_{s 6}=\left[\begin{array}{ccc}
0 & 1 & 0 \\
-1 & 0 & 0 \\
0 & 0 & 1
\end{array}\right] \text {. }
\end{aligned}
$$




\section{Data Availability}

The data used to support the findings of this study are available from the corresponding author upon request.

\section{Conflicts of Interest}

The authors indicated no potential conflicts of interest.

\section{Acknowledgments}

This work was supported by the National Key R\&D Program of China (Grant No: 2018YFC2001600) and National Natural Science Foundation of China (Grant No: 61973205).

\section{References}

[1] World Health Organization, WHO: Avoiding Heart Attacks and Strokes, Vol. 23, WHO, Geneva, Switzerland, 2005.

[2] S. Jezernik, G. Colombo, T. Keller, H. Frueh, and M. Morari, "Robotic orthosis lokomat: a rehabilitation and research tool," Neuromodulation: Technology at the Neural Interface, vol. 6, no. 2, pp. 108-115, 2003.

[3] Andago: https://www.hocoma.com/solutions/andago/.

[4] J. Patton, D. A. Brown, M. Peshkin et al., "KineAssist: design and development of a robotic overground gait and balance therapy device," Topics in Stroke Rehabilitation, vol. 15, no. 2, pp. 131-139, 2008.

[5] A. Morbi, M. Ahmadi, and A. Nativ, "GaitEnable: an omnidirectional robotic system for gait rehabilitation," in Proceedings of the International Conference on Mechatronics and Automation, August 2012.

[6] F. Firmani and E. J. Park, "A framework for the analysis and synthesis of 3D dynamic human gait," Robotica, vol. 30, no. 1, pp. 145-157, 2012.

[7] J. Ji, S. Guo, F. Xi, and L. Zhang, "Design and analysis of a smart rehabilitation walker with passive pelvic mechanism," Journal of Mechanisms and Robotics, vol. 12, no. 3, 2020.

[8] F. Xi, "Inverse kinematics of free-floating space robots with minimum dynamic disturbance," Robotica, vol. 14, no. 6, pp. 667-675, 1996.

[9] M. Vukobratovi and B. Borovac, "Zero-moment point -thirty -five years of its life," International Journal of Humanoid Robotics, vol. 1, no. 1, pp. 157-173, 2004.

[10] M. D. Vose, The Simple Genetic Algorithm, MIT Press, no. 1, pp. 31-57, Cambridge, MA, USA, 1999.

[11] C.-L. Huang and C.-J. Wang, "A GA-based feature selection and parameters optimizationfor support vector machines," Expert Systems with Applications, vol. 31, no. 2, pp. 231-240, 2006.

[12] J. Ji, S. Guo, and F. Xi, "Force analysis and evaluation of a pelvic support walking robot with joint compliance," Journal of Healthcare Engineering, vol. 2018, Article ID 9235023, 12 pages, 2018. 